\title{
Comparison of genomic predictions for lowly heritable traits using multi-step and single-step genomic best linear unbiased predictor in Holstein cattle
}

\author{
A. R. Guarini, ${ }^{\star}$ D. A. L. Lourenco,† L. F. Brito, ${ }^{\star}$ M. Sargolzaei, ${ }^{\star} \ddagger$ C. F. Baes, ${ }^{*}$ F. Miglior, ${ }^{\star} \S$ I. Misztal, $\dagger$ \\ and F. S. Schenkel ${ }^{* 1}$ \\ ${ }^{*}$ Centre for Genetic Improvement of Livestock, Department of Animal Biosciences, University of Guelph, Guelph, Ontario, Canada, N1G-2W1 \\ †Department of Animal and Dairy Science, University of Georgia, Athens 30602 \\ $\ddagger$ The Semex Alliance, Guelph, Ontario, Canada, N1H-6J2 \\ §Canadian Dairy Network, Guelph, Ontario, Canada, N1K-1E5
}

\begin{abstract}
The success and sustainability of a breeding program incorporating genomic information is largely dependent on the accuracy of predictions. For low heritability traits, large training populations are required to achieve high accuracies of genomic estimated breeding values (GEBV). By including genotyped and nongenotyped animals simultaneously in the evaluation, the singlestep genomic BLUP (ssGBLUP) approach has the potential to deliver more accurate and less biased genomic evaluations. The aim of this study was to compare the accuracy and bias of genomic predictions for various traits in Canadian Holstein cattle using ssGBLUP and multi-step genomic BLUP (msGBLUP) under different strategies, such as (1) adding genomic information of cows in the analysis, (2) testing different adjustments of the genomic relationship matrix, and (3) using a blending approach to obtain GEBV from msGBLUP. The following genomic predictions were evaluated regarding accuracy and bias: (1) GEBV estimated by ssGBLUP; (2) direct genomic value estimated by msGBLUP with polygenic effects of 5 and 20\%; and (3) GEBV calculated by a blending approach of direct genomic value with estimated breeding values using polygenic effects of 5 and $20 \%$. The effect of adding genomic information of cows in the evaluation was also assessed for each approach. When genomic information was included in the analyses, the average improvement in observed reliability of predictions was observed to be 7 and 13 percentage points for reproductive and workability traits, respectively, compared with traditional BLUP. Absolute deviation from 1 of the regression coefficient of the linear regression of de-regressed estimated breeding values on genomic predictions went from 0.19 when using
\end{abstract}

Received November 24, 2017

Accepted May 8, 2018.

${ }^{1}$ Corresponding author: schenkel@uoguelph.ca traditional BLUP to 0.22 when using the msGBLUP method, and to 0.14 when using the ssGBLUP method. The use of polygenic weight of $20 \%$ in the msGBLUP slightly improved the reliability of predictions, while reducing the bias. A similar trend was observed when a blending approach was used. Adding genomic information of cows increased reliabilities, while decreasing bias of genomic predictions when using the ssGBLUP method. Differences between using a training population with cows and bulls or with only bulls for the msGBLUP method were small, likely due to the small number of cows included in the analysis. Predictions for lowly heritable traits benefit greatly from genomic information, especially when all phenotypes, pedigrees, and genotypes are used in a single-step approach.

Key words: genomic evaluation, dairy cattle, reference population, reliability, single-step model

\section{INTRODUCTION}

In Canada, genomic evaluation for a series of traits has been successfully implemented in Holstein cattle since 2009 through a multi-step genomic BLUP method (msGBLUP; Van Doormaal et al., 2009). This procedure consists of (1) running a traditional genetic evaluation based solely on pedigree and phenotypic information, (2) calculation of pseudo-phenotypes such as de-regressed estimated breeding values (DEBV) using results obtained from the previous step, (3) estimation of SNP effects to obtain direct genomic values (DGV) for genotyped animals, and (4) blending of genomic predictions with EBV or parent averages (PA). Loss of information during the de-regression step can introduce bias and errors into the evaluation (Legarra et al., 2014), thus reducing the contribution of genomic information. Moreover, in msGBLUP, only information on genotyped animals is directly used. Given that only a small portion of the animals are genotyped, bias can also be introduced by the fact that not all information used for selection decisions is accounted for. 
To overcome potential problems, Misztal et al. (2009), Aguilar et al. (2010), and Christensen and Lund (2010) developed single-step genomic BLUP (ssGBLUP), a methodology that combines genotypic, pedigree, and phenotypic information into a single evaluation. It consists of augmenting the pedigree relationship matrix with contributions from genomic relationships into a matrix of realized (observed or imputed) relationships, the inverse of which is used in the BLUP mixed model equations. In this way, genotyped and nongenotyped animals can be included simultaneously in the evaluation. As several steps are avoided, ssGBLUP is, in many instances, simpler to use and has the potential to deliver more accurate and less biased genomic evaluations. The main advantage of ssGBLUP, especially for dairy cattle, is its ability to account for genomic preselection (Legarra et al., 2014). Accuracy of ssGBLUP is usually as high as, if not greater than, other methods of genetic merit evaluation (Christensen et al., 2012; Baloche et al., 2014; Lourenco et al., 2014a).

Accuracy of genomic predictions is critical for the expected genetic gains resulting from genomic selection and is dependent on many factors, such as heritability of the trait, the statistical method used to estimate SNP effects in the training population, and most importantly, the size of the training population (Goddard, 2009; Hayes et al., 2009; Hozé et al., 2014). For the latter, the number of genotyped progeny-tested bulls could be a limitation, especially for lowly heritable traits or those traits that are difficult or expensive to measure. One way of overcoming this problem is by incorporating genomic information of cows into the evaluation (Calus et al., 2013; Tsuruta et al., 2013; Uemoto et al., 2017). However, it is important that cow and bull traditional evaluations are comparable to avoid a decrease in reliability of genomic predictions, especially due to preferential treatment. Wiggans et al. (2011) reported a decrease in reliability of genomic predictions with the inclusion of cows in the training population. The authors hypothesized that many cows were subject to preferential treatment for having higher genetic merit. A pre-adjustment was then developed to reduce the mean and variance of cows' EBV so they would be comparable to those of bulls (Wiggans et al., 2012). Tsuruta et al. (2013) suggested that by using ssGBLUP, the inclusion of cows in the training population would be possible without any pre-adjustments.

In Canada, genomic evaluations are currently based solely on bulls' genomic information and there is still a lack of studies investigating the feasibility of ssGBLUP in Canadian dairy cattle. The incorporation of genomic information into breeding programs using alternative approaches to improve genomic predictions for lowly heritable traits is needed. In addition, the importance of workability and reproduction traits have increased over time in the dairy cattle industry. Advantages of incorporating genomics into a breeding program are the greatest for lowly heritable traits, especially due to the higher relative increase in reliabilities of predictions achieved for these traits by using genomic information (García-Ruiz et al., 2016; Wiggans et al., 2017). Therefore, the objective of this study was to compare the accuracy and bias (spread) of genomic predictions for various workability and reproductive traits in Canadian Holstein cattle using multi-step and single-step GBLUP methods under different strategies, such as adding genomic information of cows in the analysis, testing different adjustments of the genomic relationship matrix, and using a blending approach to obtain GEBV from the multi-step method.

\section{MATERIALS AND METHODS}

\section{Data}

Genotypic data consisted of 10,590 bulls born between 1960 and 2012 genotyped with the Illumina Bovine SNP50 BeadChip (50K, Illumina Inc., San Diego, CA) or a higher density panel. The SNP present in the higher density panel that overlapped with the $50 \mathrm{~K}$ panel were imputed to the $50 \mathrm{~K}$ panel using the FImpute software (Sargolzaei et al., 2014). In addition, 6,842 cows born between 1997 and 2015 genotyped either with the 50K SNP panel or a low-density panel (6K) were imputed to the $50 \mathrm{~K}$ panel by also using the FImpute software. Thus, all animals had information on the same SNP set. Genotypes were coded as 0, 1, or 2 for calculation of the genomic relationship matrix $(\mathbf{G})$. Genotype quality control excluded monomorphic SNP, SNP and individuals with call rate lower than $90 \%$, SNP that were out of Hardy-Weinberg equilibrium with very low probability $\left(P<10^{-6}\right)$ or with minor allele frequency less than 0.05 , and individuals with parentprogeny Mendelian conflicts. After quality control, the number of genotyped animals retained was 17,430 and the final genotype data set included 40,635 informative SNP. The PREGSF90 software was used for SNP and sample quality control (Misztal et al., 2002).

Data on Holstein reproductive and workability traits were extracted from the April 2017 genetic evaluation carried out by the Canadian Dairy Network (Guelph, Ontario, Canada, www.cdn.ca). The traits selected for this study were milking speed (MS), milking temperament (MT), age at first insemination (AFS), days from calving to first insemination (CTFS), number of services (NS), 56-d nonreturn rate (NRR), days from first service to conception (FSTC), calving ease (CE), stillbirth (SB), gestation length $(\mathbf{G L})$, and calf size 
(CZ). Traits that were recorded during or before first calving were considered as heifer traits and were coded as parity 0 . Traits measured in first-lactation cows were coded as parity 1 and the same approach was applied to subsequent lactations (parity $>1$ ). Single-trait analyses were carried out for all heifer and cow traits. The animal models used for genetic evaluation can be found in Jamrozik and Kistemaker (2016). Descriptive statistics for each trait are shown in Table 1.

De-regressed EBV were used as response variables for genomic prediction in the msGBLUP approach. De-regression was computed following VanRaden et al. (2009):

$$
\begin{gathered}
\mathrm{ERC}_{\mathrm{P}}=\left[\lambda \frac{\mathrm{EBV}_{\text {rel }}}{\left(1-\mathrm{EBV}_{\mathrm{rel}}\right)}\right]-\mathrm{ERC}_{\mathrm{PA}}, \\
\mathrm{ERC}_{\mathrm{PA}}=\lambda \frac{\mathrm{PA}_{\text {rel }}}{\left(1-\mathrm{PA}_{\mathrm{rel}}\right)}, \\
\mathrm{DEBV}=\mathrm{PA}+\left[\frac{\mathrm{EBV}-\mathrm{PA}}{\mathrm{ERC}_{\mathrm{P}} /\left(\mathrm{ERC}_{\mathrm{P}}+\mathrm{ERC}_{\mathrm{PA}}+1\right)}\right],
\end{gathered}
$$

where DEBV are de-regressed breeding values, $\mathrm{ERC}_{\mathrm{PA}}$ is the effective record contribution from $\mathrm{PA}, \mathrm{ERC}_{\mathrm{P}}$ is the effective record contribution from own record/ progeny information, $\mathrm{PA}_{\text {rel }}$ is reliability of parent average, $\lambda$ is equal to $\left(1-h^{2}\right) / h^{2}$, and $E B V_{\text {rel }}$ is reliability of EBV. For computational ease, de-regressed EBV of bulls were not adjusted for the potential contributions from daughters in the training population because the average number of daughters per bull in the training set was very small $(\mathrm{n} \sim 0.10)$ compared with the minimum number of daughters required for a bull to be included in the training set $(n=10)$. Therefore, most of the bull information came from daughters not included in the training set, making the double counting of information very small. In scenarios with a much larger number of cows included in the training population relative to the number of bulls, where bulls would be expected to have several daughters in the training population, an adjustment to prevent double counting would be necessary, such as the iterative procedure proposed by Calus et al. (2016).

Lourenco et al. (2014b) have shown that by retaining only 4 to 5 generations of pedigree records decreased computing costs when using ssGBLUP approach and did not significantly decrease the accuracy of genomic evaluations. Therefore, the pedigree file used here for the analysis was generated by tracing the pedigree of animals with records for each trait to 4 generations back and its size ranged from 2,343,158 animals for

\begin{tabular}{|c|c|c|c|c|c|c|c|}
\hline Parity & Trait $^{1}$ & $h^{2}$ & No. of records & Mean & $\mathrm{SD}$ & Minimum & Maximum \\
\hline \multicolumn{8}{|l|}{ Reproductive trait } \\
\hline \multirow[t]{8}{*}{ First } & AFS & 0.09 & $1,217,701$ & 478.97 & 55.81 & 274 & 639 \\
\hline & NRR & 0.03 & $1,404,619$ & 0.72 & 0.45 & 0 & 1 \\
\hline & NS & 0.02 & $1,420,060$ & 1.62 & 1.04 & 1 & 10 \\
\hline & FSTC & 0.03 & $1,086,405$ & 18.25 & 33.64 & 0 & 206 \\
\hline & GL & 0.19 & $1,000,420$ & 278.78 & 6.41 & 240 & 300 \\
\hline & $\mathrm{CE}$ & 0.12 & $1,075,704$ & 1.57 & 0.68 & 1 & 4 \\
\hline & $\mathrm{SB}$ & 0.11 & $1,068,520$ & 0.89 & 0.31 & 0 & 1 \\
\hline & $\mathrm{CZ}$ & 0.11 & $1,040,971$ & 2.01 & 0.57 & 0 & 3 \\
\hline \multirow[t]{8}{*}{ Later } & NRR & 0.04 & $3,771,204$ & 0.55 & 0.50 & 0 & 1 \\
\hline & NS & 0.06 & $3,815,035$ & 2.31 & 1.62 & 1 & 10 \\
\hline & CTFS & 0.07 & $3,803,907$ & 86.95 & 31.54 & 14 & 244 \\
\hline & FSTC & 0.08 & $2,545,203$ & 37.34 & 47.98 & 0 & 206 \\
\hline & $\mathrm{GL}$ & 0.16 & $2,359,727$ & 279.88 & 6.54 & 240 & 300 \\
\hline & $\mathrm{CE}$ & 0.08 & $2,747,813$ & 1.40 & 0.59 & 1 & 4 \\
\hline & SB & 0.01 & $2,733,684$ & 0.95 & 0.22 & 0 & 1 \\
\hline & $\mathrm{CZ}$ & 0.09 & $2,704,295$ & 2.19 & 0.58 & 0 & 3 \\
\hline \multicolumn{8}{|l|}{ Workability trait } \\
\hline \multirow[t]{2}{*}{ First $^{2}$} & MT & 0.13 & $2,921,775$ & 3.29 & 0.75 & 1 & 5 \\
\hline & MS & 0.14 & $3,573,346$ & 3.12 & 0.70 & 1 & 5 \\
\hline
\end{tabular}
CTFS to 5,070,448 for MS.

Table 1. Descriptive statistics and $\mathrm{h}^{2}$ of reproductive and workability traits in first and later parities

${ }^{1}$ Age at first service (AFS) in days, calf size (CZ), calving ease (CE), days from calving to first service (CTFS), days from first service to conception (FSTC), gestation length (GL) in days, milking speed (MS), milking temperament (MT), 56-d nonreturn rate (NRR), number of services (NS), stillbirth (SB).

${ }^{2}$ For both MS and MT, scoring is made only once during the first 6 mo of first lactation. 


\section{Statistical Models}

The statistical models used for the analysis in different scenarios are described below.

Multi-Step GBLUP Model. This approach consisted of 4 steps:

(1) Estimation of breeding values using traditional BLUP;

(2) De-regression of EBV;

(3) Prediction of DGV; and

(4) Blending of DGV with PA/EBV.

Consider the following model:

$$
\mathbf{y}=1 \mu+\mathrm{Zg}+\mathbf{e}
$$

where $\mathbf{y}$ is a vector of de-regressed evaluations of genotyped animals, $\mathbf{1}$ is a vector of ones, $\mu$ is the overall mean, $\mathbf{Z}$ is a design matrix that allocates records to breeding values, $\mathbf{g}$ is a vector of DGV to be estimated, and $\mathbf{e}$ is a vector of random residual effects. It was assumed that $\mathrm{g} \sim N\left(\mathbf{0}, \mathbf{G} \sigma_{\mathrm{g}}^{2}\right)$, where $\mathbf{G}$ is the genomic relationship matrix based on SNP markers, and $\sigma_{\mathrm{g}}^{2}$ is the additive genetic variance; e $\sim N\left(\mathbf{0}, \mathbf{R} \sigma_{\mathrm{e}}^{2}\right)$, where $\mathbf{R}$ is a diagonal matrix containing weights based on the reliability of the breeding values and $\sigma_{\mathrm{e}}^{2}$ is the residual variance.

Here, DGV were estimated with either 5 or $20 \%$ weight $(\delta)$ for the expected additive relationship matrix when combined with the genomic relationship matrix. Thus, the model becomes

$$
\mathbf{y}=1 \mu+\mathbf{Z g}_{\delta}+\mathbf{e},
$$

where $\mathbf{g}_{\delta} \sim N\left(\mathbf{0}, \mathbf{G}_{\delta} \sigma_{\mathrm{g}_{\delta}}^{2}\right)$, where $\mathbf{G}_{\delta}$ is a combined relationship matrix, $\mathbf{G}_{\delta}=\delta \mathbf{A}_{22}+(1-\delta) \mathbf{G}$.

Blending. The blending approach used to generate GEBV was as follows:

$$
\begin{aligned}
\mathrm{GEBV} & =\mathrm{w}_{1} \mathrm{DGV}+\mathrm{w}_{2} \mathrm{EST}, \\
\mathrm{w}_{1} & =\frac{\operatorname{Rel}_{\mathrm{DGV}}}{\operatorname{Rel}_{\mathrm{DGV}}+\operatorname{Rel}_{\mathrm{EST}}}, \\
\mathrm{w}_{2} & =\frac{\operatorname{Rel}_{\mathrm{EST}}}{\operatorname{Rel}_{\mathrm{EST}}+\operatorname{Rel}_{\mathrm{DGV}}},
\end{aligned}
$$

where EST is either EBV (for training population animals) or PA (for validation population animals) from traditional BLUP, and Rel is the reliability of estimates.
Single-Step GBLUP Model. This method uses information from both genotyped and nongenotyped animals simultaneously. The mixed model equations then become

$$
\left[\begin{array}{cc}
\mathbf{X}^{\prime} \mathbf{X} & \mathbf{X}^{\prime} \mathbf{Z} \\
\mathbf{Z}^{\prime} \mathbf{X} & \mathbf{Z}^{\prime} \mathbf{Z}+\mathbf{H}^{-1} \alpha
\end{array}\right]\left[\begin{array}{l}
\mathbf{b} \\
\mathbf{u}
\end{array}\right]=\left[\begin{array}{l}
\mathbf{X}^{\prime} \mathbf{y} \\
\mathbf{Z}^{\prime} \mathbf{y}
\end{array}\right]
$$

where $\mathbf{y}$ is the vector of observations, $\mathbf{b}$ is the vector of fixed effects, $\mathbf{u}$ is the vector of random additive genetic effects, $\mathbf{X}$ and $\mathbf{Z}$ are the corresponding design matrices, $\alpha$ is the variance ratio, and $\mathbf{H}^{-1}$ is derived as in Aguilar et al. (2010) and Christensen and Lund (2010):

$$
\mathbf{H}^{-1}=\mathbf{A}^{-1}+\left[\begin{array}{cc}
0 & 0 \\
0 & \left(\mathbf{G}^{-1}-\omega \mathbf{A}_{22}^{-1}\right)
\end{array}\right],
$$

where $\mathbf{A}$ is the pedigree relationship matrix, $\mathbf{G}$ is the genomic relationship matrix, as $0.95 \mathbf{G}^{*}+0.05 \mathbf{A}_{22}$ (to avoid singularity problems), and $\mathbf{G}^{*}$ is constructed as in VanRaden (2008); $\mathbf{A}_{22}$ is the pedigree relationship matrix among genotyped animals. Inbreeding was considered in the construction of all the relationship matrices. Differences between $\mathbf{A}_{22}$ and $\mathbf{G}$ may occur for several reasons that should be taken into account. For instance, incompleteness and shortness of pedigree, pedigree mistakes, incorrect assignment of genotypes, and poor quality of genotypes can cause such differences resulting in upward bias of genomic EBV for young animals and poor convergence when iteratively solving the mixed model equations for ssGBLUP (Misztal et al., 2013). Previous studies have investigated the use of a scaling factor or weight $(\omega)$ on $\mathbf{A}_{22}^{-1}$ to avoid convergence problems and bias, and it has been reported that values lower than 1 usually contribute to reduce the bias of GEBV for various traits in dairy cattle (Misztal et al., 2010; Tsuruta et al., 2011). Therefore, a weight $(\omega)$ ranging from 1.0 to 0.6 for different traits was also applied to $\mathbf{A}_{22}^{-1}$ in this study $\left(\omega=1\right.$ for $\mathrm{FSTC}_{\mathrm{h}}, \mathrm{SB}_{\mathrm{h}}$, $\mathrm{NRR}_{\mathrm{c}}$, and $\mathrm{FSTC}_{\mathrm{c}}$, where subscript $\mathrm{h}$ refers to heifer traits and c refers to cow traits; $\omega=0.8$ for CZ; $\omega=0.7$ for $\mathrm{NS}_{\mathrm{c}}, \mathrm{GL}_{\mathrm{c}}$, and $\mathrm{MS}$; and $\omega=0.6$ for $\mathrm{AFS}, \mathrm{NRR}_{\mathrm{h}}$, $\mathrm{NS}_{\mathrm{h}}, \mathrm{GL}_{\mathrm{h}}, \mathrm{CE}_{\mathrm{h}}$, CTFS, $\mathrm{CE}_{\mathrm{c}}, \mathrm{SB}_{\mathrm{c}}, \mathrm{CZ}_{\mathrm{c}}$, and $\mathrm{MT}$ ). All models described above were also used for analyses including (or not including) cows' genomic information.

\section{Genomic Predictions}

The following genomic predictions were evaluated with regarding accuracy and bias: GEBV estimated by ssGBLUP (ssGEBV); DGV estimated by msGB- 
LUP (hereafter called the $\mathbf{G B L U P}_{\mathrm{D}}$ ) with either $5 \%$ weight for the expected additive relationship matrix of genotyped individuals $\left(\mathbf{A}_{\mathbf{2 2}}\right)$ when combined with the genomic relationship matrix $(\mathbf{D G V}-\mathbf{5 \%} \mathbf{A})$ or $20 \%$ weight (DGV-20\%A); and GEBV calculated by blending traditional EBV/PA with DGV with either $5 \%(\mathbf{G E B V}-\mathbf{5 \%} \mathbf{A})$ or $20 \%(\mathbf{G E B V}-\mathbf{2 0 \%} \mathbf{A})$ weight for $\mathbf{A}_{\mathbf{2 2}}$ (hereafter called the $\mathbf{G B L U P}_{\mathbf{B}}$ method). In addition, all analyses were carried out including or not including the genomic information of cows. Only cows with DEBV reliabilities equivalent to the reliability due to the contribution of at least 10 daughters were considered in the training population. The weights of 5 and $20 \%$ for expected additive relationship matrix were chosen because in the Canadian dairy cattle official genomic evaluations a weight of $20 \%$ has been defined and is used (Van Doormaal et al., 2009), whereas in other countries the weight usually ranges between 5 and 20\%. Genomic predictions using the GBLUP method were computed using the gebv software (Sar- golzaei et al., 2009) for the msGBLUP method and BLUP90IOD2 program (Tsuruta et al., 2001) for the ssGBLUP method.

For this study, different data sets were prepared to assess prediction performance for the traits of interest: (1) a full data set containing all records of cows from which EBV were calculated and de-regressed to be used as benchmark; and (2) a reduced data set that included records of cows after deleting the last $4 \mathrm{yr}$ of observations. These 2 data sets were used for genomic predictions as previously described. Subsequently, animals from the reduced data set were split into training and validation populations according to year of birth. The validation set (used to assess GEBV prediction reliability and bias) included only genotyped bulls with no daughters in the reduced data set, but with at least 50 daughters in the full data set. Table 2 summarizes the number of bulls and cows in each data set, as well as the average reliability of DEBV for the msGBLUP method for each trait.

Table 2. Structure of training and validation data sets for multi-step genomic BLUP (msGBLUP) and single-step genomic BLUP (ssGBLUP) methods

\begin{tabular}{|c|c|c|c|c|c|c|c|c|c|}
\hline \multirow[b]{4}{*}{ Parity } & \multirow[b]{4}{*}{ Trait $^{1}$} & \multirow{4}{*}{\multicolumn{2}{|c|}{$\begin{array}{c}\begin{array}{c}\text { Validation } \\
\text { population }\end{array} \\
\text { Birth year }\end{array}$}} & \multicolumn{6}{|c|}{ Training population } \\
\hline & & & & \multicolumn{4}{|c|}{ msGBLUP } & \multicolumn{2}{|c|}{ ssGBLUP } \\
\hline & & & & \multicolumn{2}{|c|}{ Bulls } & \multicolumn{2}{|c|}{ Cows } & \multirow[b]{2}{*}{ Phenotype $^{5}$} & \multirow{2}{*}{$\begin{array}{c}\text { Phenotype } \\
\text { and } \\
\text { genotype }^{5}\end{array}$} \\
\hline & & & & $\mathrm{N}^{3}$ & $\begin{array}{c}\operatorname{Rel}_{\text {DEBV }}{ }^{4} \\
(\%)\end{array}$ & $\mathrm{N}$ & $\begin{array}{c}\operatorname{Rel}_{\text {DEBV }} \\
(\%)\end{array}$ & & \\
\hline \multicolumn{10}{|c|}{ Reproductive trait } \\
\hline \multirow{7}{*}{ First } & NRR & 317 & 2009-2011 & 4,785 & 22 & 669 & 04 & $1,004,755$ & 5,796 \\
\hline & NS & 241 & 2009-2011 & 5,146 & 29 & 743 & 03 & $1,035,548$ & 5,818 \\
\hline & FSTC & 209 & $2008-2010$ & 4,126 & 17 & 305 & 04 & 774,808 & 5,551 \\
\hline & GL & 418 & 2009-2011 & 5,111 & 68 & 723 & 18 & 761,889 & 5,018 \\
\hline & $\mathrm{CE}$ & 438 & 2009-2011 & 5,359 & 65 & 788 & 16 & 825,001 & 5,119 \\
\hline & SB & 438 & 2009-2011 & 5,330 & 44 & 660 & 07 & 817,817 & 5,118 \\
\hline & $\mathrm{CZ}$ & 420 & 2009-2011 & 5,316 & 58 & 711 & 12 & 801,544 & 5,024 \\
\hline \multirow{3}{*}{ Later } & $\mathrm{CE}$ & 363 & $2008-2010$ & 6,089 & 65 & 407 & 29 & $2,422,682$ & 5,886 \\
\hline & SB & 285 & $2008-2010$ & 6,040 & 28 & 399 & 06 & $2,408,553$ & 5,886 \\
\hline & $\mathrm{CZ}$ & 350 & 2008-2010 & 6,060 & 67 & 405 & 32 & $2,387,080$ & 5,872 \\
\hline \multicolumn{10}{|c|}{ Workability trait } \\
\hline \multirow[t]{2}{*}{ First $^{6}$} & MT & 554 & 2009-2011 & 7,047 & 74 & 772 & 13 & $2,485,983$ & 6,184 \\
\hline & MS & 554 & 2009-2011 & 7,369 & 76 & 779 & 15 & $3,136,478$ & 6,150 \\
\hline
\end{tabular}

${ }^{1}$ Age at first service (AFS), calf size (CZ), calving ease (CE), days from calving to first service (CTFS), days from first service to conception (FSTC), gestation length (GL), milking speed (MS), milking temperament (MT), 56-d nonreturn rate (NRR), number of services (NS), stillbirth (SB).

${ }^{2}$ Only bulls with at least 50 daughters were considered in the validation population.

${ }^{3}$ Only bulls with at least 10 daughters were considered in the training population.

${ }^{4}$ Average reliability of de-regressed EBV in the training population.

${ }^{5}$ Number of animals with phenotypes and number of animals with both phenotypes and genotypes.

${ }^{6}$ For both MS and MT, scoring is made only once during the first 6 mo of first lactation. 


\section{Validation}

The reliability of genomic predictions for the studied traits were measured as squared correlation between the predicted breeding values (obtained with the reduced data set) and de-regressed EBV (from the full data set) in the validation data sets. To access the bias (spread) of genomic predictions for each method, the following regression model was used:

$$
\operatorname{DEBV}_{\mathrm{C}}=\mathrm{b}_{0}+\mathrm{b}_{1} \mathrm{X}_{\mathrm{P}}+\mathrm{e},
$$

where $\mathrm{DEBV}_{\mathrm{C}}$ are de-regressed EBV, obtained from the complete data set, of genotyped bulls with no daughters in the reduced data set, but with at least 50 daughters in the complete data set; $b_{0}$ is the intercept; $\mathrm{b}_{1}$ is the linear regression coefficient indicating bias of the predictions; $\mathrm{X}_{\mathrm{P}}$ is the bull's PA or GEBV obtained with the reduced data set; and e is the residual.

\section{RESULTS AND DISCUSSION}

In the present study, when genomic information was included in the analyses, the average improvement in observed reliability of predictions was 7 and 13 percentage points for reproductive traits and workability traits, respectively, compared with traditional BLUP (Table 3). Moreover, averaged over the 18 traits, the absolute deviation from 1 of the regression coefficient of the linear regression of de-regressed EBV on genomic predictions went from 0.19 when using traditional BLUP to 0.22 when using msGBLUP, and to 0.14 when using ssGBLUP (Table 4). Regression coefficients ranged from 0.51 to 1.35 for msGBLUP, whereas for ssGBLUP, the coefficients ranged from 0.66 to 1.04 (Table 4).

Regression coefficient values close to 1 suggest that 1-unit difference in the genomic evaluation results in a 1-unit change in the trait, and therefore, they are considered to be on the same scale (Sullivan, 2009; Wiggans et al., 2011). A regression coefficient less than 1 indicates inflation of the variance of genomic predictions, whereas deflation occurs when the coefficient is larger than 1. Traditional BLUP models consider all information available in the evaluation (i.e., phenotypes and pedigree), and therefore, less bias derived from selection is observed (Sorensen and Kennedy, 1984). Bias on multi-step genomic predictions may arise from the fact that genomic prediction models do not simultaneously fit all data used for selection decisions, and therefore, it is assumed that the populations being evaluated have not undergone selection. Yet, in dairy cattle, a large number of genotyped populations usually consist of progeny-tested bulls, which are highly selected animals (Hayes et al., 2009; Vitezica et al., 2011).
By including all information available in the evaluation simultaneously (i.e., phenotypes, genotypes, and pedigree), ssGBLUP is able to partially account for pre-selection, thus minimizing possible selection bias on the predictions (Legarra et al., 2014). In addition, there are various specificities in msGBLUP regarding PA information (VanRaden et al., 2009), whereas in the ssGBLUP PA are automatically included in the genomic predictions. Gao et al. (2012) applied 3 multistep GBLUP and 2 single-step blending methods for genomic prediction in Nordic Holsteins. In their study, the 2 single-step methods led to less bias than the other GBLUP methods. Similarly, Ma et al. (2015) showed that regression coefficients went from 0.69 when using a multi-step GBLUP to 0.78 for the single-step GBLUP method. In the present study, averaged over all traits, ssGBLUP resulted in regression coefficients with the smallest absolute deviation from 1. For reproductive and workability traits, the deviation of the regression coefficient from 1 for ssGBLUP was equal to 0.11 and 0.19 , respectively, whereas for msGBLUP, this deviation was 0.21 and 0.25 , respectively. The single-step approach also resulted in a slight increase in reliability of genomic predictions compared with those of msGBLUP.

Historically, ssGBLUP could not converge or had a large bias in some cases, and one measure to improve on both counts was a weight parameter $(\omega)$ given to $\mathbf{A}_{22}^{-1}$. Such a parameter was shown to account for missing inbreeding, especially when some pedigrees were missing (Misztal et al., 2017). When inbreeding was accounted for in $\mathbf{A}$ including in phantom parent groups, the $b_{1}$ parameter improved from 0.75 to 0.90 . Accounting for reduced heritability due to genetic selection improved the parameter $b_{1}$ to 0.96 and slightly increased accuracy. These measures indicated that special modeling is required to account for selection practices under genomic selection (Misztal et al., 2017).

Obtaining unbiased predictions is of fundamental importance for an accurate ranking and fair comparison of animals across generations (Patry and Ducrocq, 2009; Aguilar et al., 2010). Gao et al. (2012) showed that absolute deviations of the regression coefficients from 1 tended to decrease with increasing polygenic weights in a GBLUP model, which is consistent with the literature (Aguilar et al., 2010; Liu et al., 2011). This could be explained by the fact that genetic markers are not able to explain the total genetic variance of a given trait. Thus, the polygenic effect would account for the residual genetic variance, which is not accounted for by using only genetic markers. In the present study, the polygenic weight of $20 \%$ in the msGBLUP slightly improved the observed reliability of predictions while reducing the bias. Averaged over all traits, the deviation of the regression coefficient from 1 for GBLUP $_{D}$ 
GUARINI ET AL.

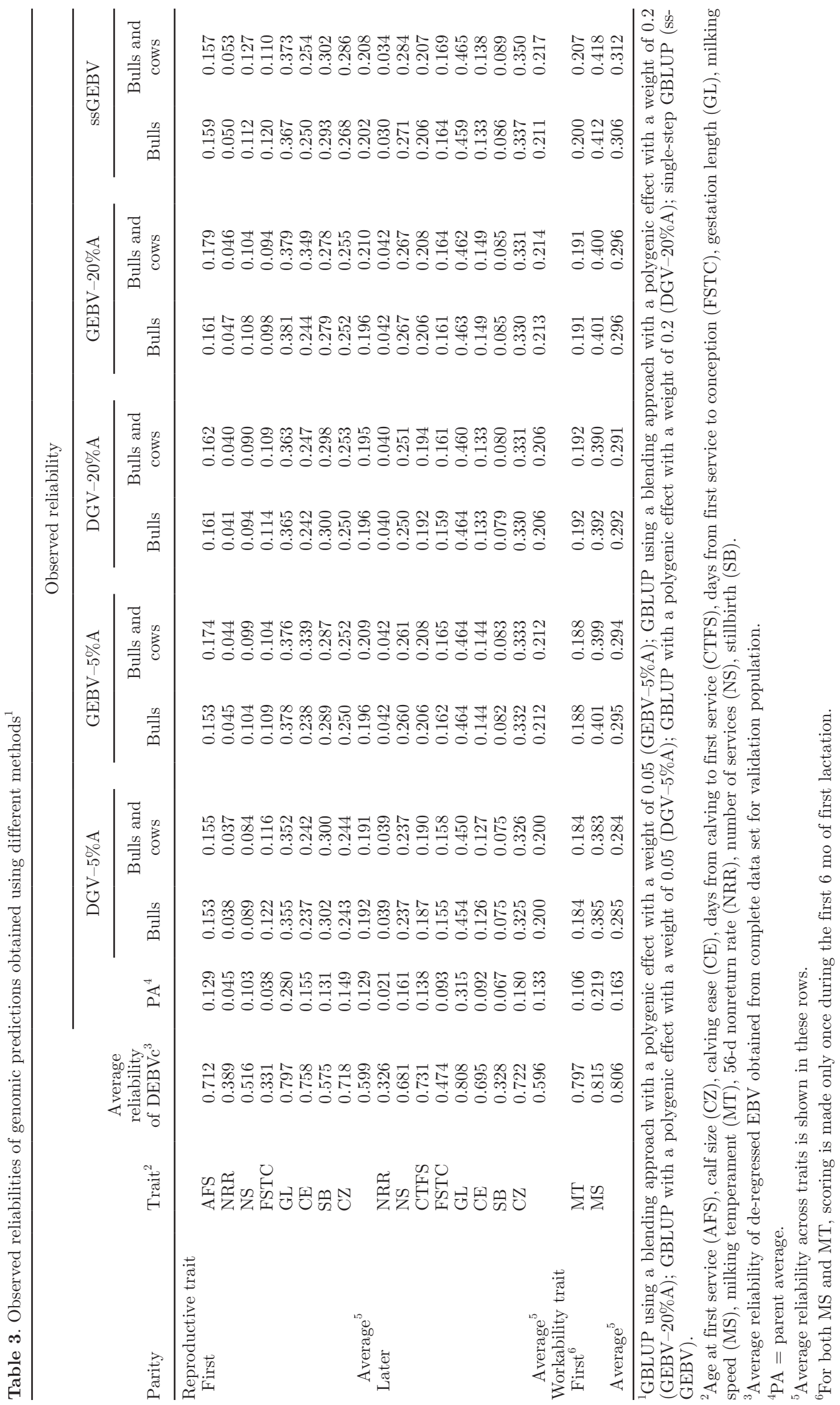


GENOMIC PREDICTIONS IN DAIRY CATTLE

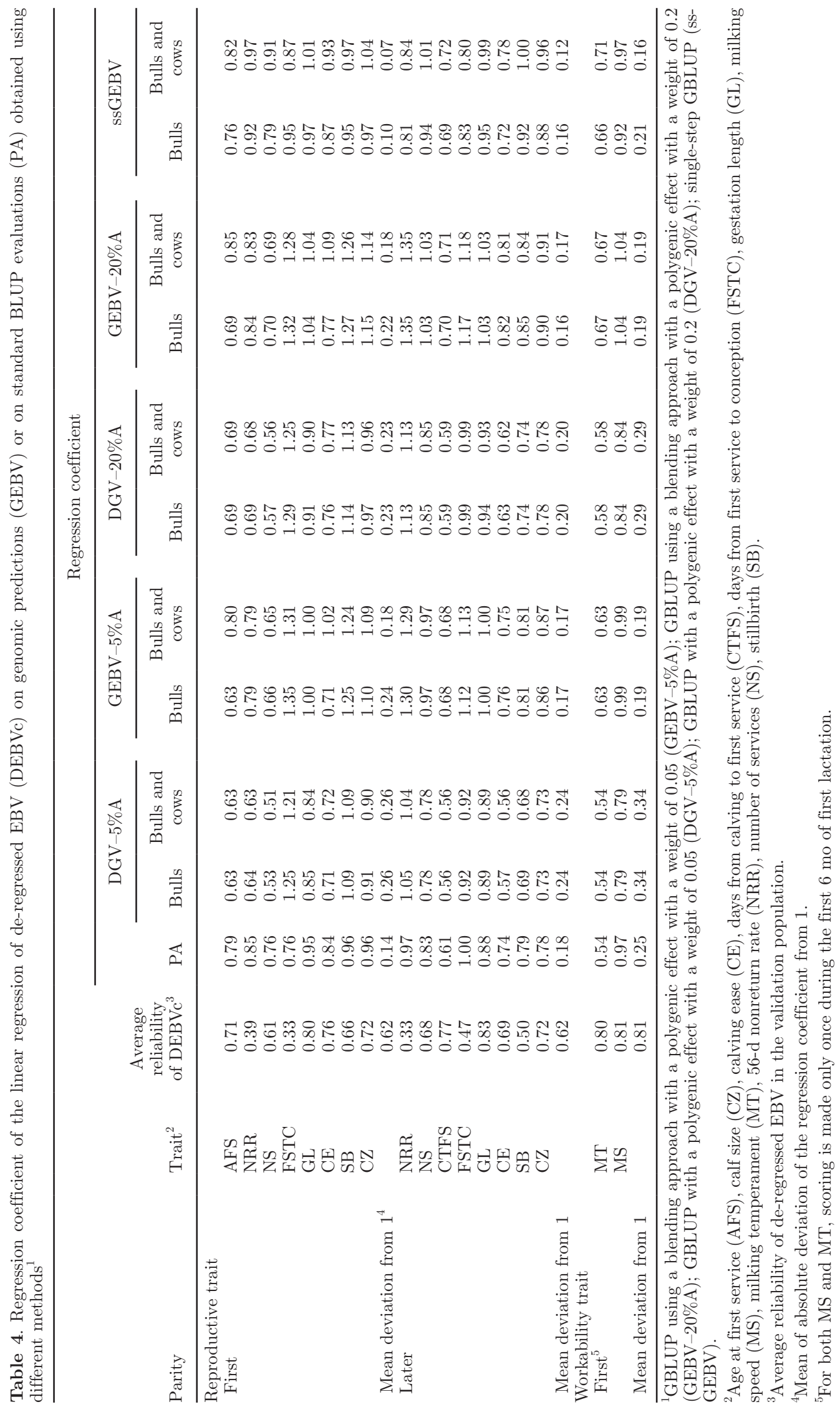


went from 0.28 when using a $5 \%$ polygenic weight to 0.24 with a $20 \%$ polygenic weight.

Alternatively, one way to account for the residual genetic variance and ensure that all polygenes are considered is by adopting a blending approach (Sullivan, 2009). In Canada, when using this blending approach, GEBV are calculated by combining EBV obtained from traditional pedigree-based (BLUP) evaluations with DGV from GBLUP evaluations through a selection index, weighted by their reliabilities (Van Doormaal et al., 2009). Predictions obtained with GBLUP ${ }_{B}$ were, on average, less biased and slightly more reliable than those obtained with GBLUP $_{D}$. When a polygenic weight of $5 \%$ was used, reliabilities ranged from 0.037 to 0.454 (average: 0.206 ) and from 0.042 to 0.464 (average: 0.217 ), for GBLUP $_{D}$ and GBLUP $_{B}$, respectively, whereas the range went from 0.040 to 0.464 (average: 0.21 ) and from 0.042 to 0.463 (average: 0.218 ), for GBLUP $_{\mathrm{D}}$ and GBLUP $_{\mathrm{B}}$, respectively, when using $20 \%$ polygenic weight (Table 3). These results are in agreement with Lourenco et al. (2014). The authors reported higher reliabilities for GEBV compared with DGV for milk yield, fat, and protein percentages. Conversely, $\mathrm{Su}$ et al. (2012) reported higher reliabilities for DGV compared with ssGEBV for various traits of Nordic Red Cattle. However, according to the authors the reliability of DGV could be overestimated, given the fact that different scaling factors were used as weights for the genomic relationship matrices in each method.

In the present study, in addition to the increase in reliability caused by blending $\left(\mathrm{GBLUP}_{\mathrm{B}}\right)$, the GEBV bias was reduced. For a polygenic weight of $5 \%$, the deviation of the regression coefficient from 1 was 0.28 and 0.19 for GBLUP $_{D}$ and GBLUP $_{\mathrm{B}}$, respectively, whereas for predictions obtained with a $20 \%$ polygenic weight, this deviation was 0.24 for GBLUP $_{\mathrm{D}}$ and 0.18 for $\operatorname{GBLUP}_{\mathrm{B}}$ (Table 4). Commonly, only animals selected based on their GEBV will have their progeny's phenotypes recorded. However, such information is not included in the evaluation, and therefore, pre-selection is not properly accounted for; this will lead to biased pedigree-based predictions (i.e., EBV; Patry and Ducrocq, 2011) and, consequently, affect the blending approach. The use of all information available simultaneously to predict GEBV could potentially help avoid such bias.

In dairy cattle genomic evaluation, training populations are usually composed of progeny-tested bulls, as those have more reliable information. However, for lowly heritable traits, such as health and fertility, a very large training population is required to achieve high accuracies of predictions (Hayes et al., 2009), which could be a challenge given the fact that progenytested bulls are limited for those traits. Moreover, progeny-tested bulls are intensively selected, which could potentially lead to loss of genetic variability. The inclusion of cows in training populations is appealing, as they can contribute to increase the size of a training population and are also of major importance during the selection decision process (Wiggans et al., 2011; Buch et al., 2012; Tsuruta et al., 2013; Ma et al., 2015). In this study, the method used for de-regression of EBV was the one proposed by VanRaden et al. (2009), due to its easy and efficient implementation. To our best knowledge, there is no consensus on the best method to use for the traits analyzed here. However, other more computationally demanding alternatives have also been proposed, as for instance in Jairath et al. (1998) and Garrick et al. (2009).

The benefits of adding cows' genomic information into the training populations may depend on the strategy adopted (Tsuruta et al., 2013; Gao et al., 2015). When using a multi-step approach, accuracies of genomic predictions are contingent on the accuracies of de-regressed EBV used and for the cows' EBV lower accuracies are expected compared with those of bulls. In the present study, differences between using a training population with cows and bulls or with bulls only for the msGBLUP methods were small (Tables 3 and 4). This is likely due to the small number of cows included in the analyses and their low reliabilities (Table 2). However, for ssGBLUP the inclusion of cows' information had a positive effect on genomic predictions. Across all traits, the single-step approach including bulls and cows' genomic information led to the highest reliability of genomic predictions, followed by ssGBLUP with bulls only in the training population, and msGBLUP methods. On average, reliabilities of ssGBLUP including bulls and cows' genomic information were $1.21 \%$ higher than those of msGBLUP methods with a bullsonly training population, which is the method currently used for Canadian genomic evaluations. Tsuruta et al. (2013) also reported an increase in reliability of 1.9 to $2.7 \%$ when cow genotypes were added in the evaluation using ssGBLUP.

The inclusion of cows' genomic information can also help reduce the bias of genomic predictions under a single-step approach. In the present study, the average deviation of the regression coefficient from 1 was 0.18 and 0.19 for GBLUP ${ }_{B}$ for bulls and cows, and bulls only in the training population, respectively. However, when using the single-step approach this deviation decreased to 0.12 when bulls and cows' genomic information was used for the analysis and when only bulls' genomic information was used, the average deviation across all traits was 0.16 . The use of a single-step approach also resulted in a slightly increased mean reliability $(0.221)$ compared with GBLUP B $_{\mathrm{B}}(0.217)$. 
The average deviation from 1 for msGBLUP was 0.22 and 0.25 for reproductive and workability traits, respectively, when only bulls were included in the training, whereas for ssGBLUP the deviation from 1 was 0.13 and 0.21 for reproductive and workability traits, respectively. When both cows and bulls were included in the training population the average deviation for msGBLUP was 0.20 and 0.25 for reproductive and workability traits, respectively, and was 0.09 and 0.16 for ssGBLUP, respectively. The results obtained for regression coefficients are in agreement with those of previous findings (Tsuruta et al., 2013; Ma et al., 2015; Uemoto et al., 2017).

\section{CONCLUSIONS}

Single-step genomic BLUP can reduce bias while slightly increasing observed reliability of genomic predictions. Predictions obtained with the singlestep approach by adding cows' genomic information showed a small gain in reliability and a reduction in bias compared with those obtained by only including bulls' genomic information. For the multi-step GBLUP method, no effects on reliability or bias of predictions were observed when genomic information of cows was considered. This may be explained by the fact that only a small number of cows were included in the analyses. The inclusion of a polygenic effect in the multi-step analysis as well as the use of a blending approach had a small effect on reliabilities of predictions, but an important effect on bias. However, the improvements obtained with different strategies adopted in the multistep method were smaller compared with the use of the single-step approach.

\section{ACKNOWLEDGMENTS}

This research was supported in main part by Agriculture and Agri-Food Canada and by additional contributions from Dairy Farmers of Canada, the Canadian Dairy Network, and the Canadian Dairy Commission under the Agri-Science Clusters Initiative. The first author is also thankful to CAPES (Brazilian Federal Agency for Support and Evaluation of Graduate Education, Brasilia, Brazil) for financial support.

\section{REFERENCES}

Aguilar, I., I. Misztal, D. L. Johnson, A. Legarra, S. Tsuruta, and T. J. Lawlor. 2010. Hot topic: a unified approach to utilize phenotypic, full pedigree, and genomic information for genetic evaluation of Holstein final score. J. Dairy Sci. 93:743-752. https://doi.org/10 .3168/jds.2009-2730.

Baloche, G., A. Legarra, G. Sallé, H. Larroque, J.-M. Astruc, C. Robert-Granié, and F. Barillet. 2014. Assessment of accuracy of genomic prediction for French Lacaune dairy sheep. J. Dairy Sci. 97:1107-1116. https://doi.org/10.3168/jds.2013-7135.

Buch, L. H., M. Kargo, P. Berg, J. Lassen, and A. C. Sørensen. 2012. The value of cows in reference populations for genomic selection of new functional traits. Animal 6:880-886. https://doi.org/10.1017/ S1751731111002205.

Calus, M. P. L., Y. de Haas, and R. F. Veerkamp. 2013. Combining cow and bull reference populations to increase accuracy of genomic prediction and genome-wide association studies. J. Dairy Sci. 96:6703-6715. https://doi.org/10.3168/jds.2012-6013.

Calus, M. P. L., J. Vandenplas, J. ten Napel, and R. F. Veerkamp. 2016. Validation of simultaneous deregression of cow and bull breeding values and derivation of appropriate weights. J. Dairy Sci. 99:6403-6419. https://doi.org/10.3168/jds.2016-11028.

Christensen, O. F., and M. S. Lund. 2010. Genomic prediction when some animals are not genotyped. Genet. Sel. Evol. 42:2. https:// doi.org/10.1186/1297-9686-42-2.

Christensen, O. F., P. Madsen, B. Nielsen, T. Ostersen, and G. Su. 2012. Single-step methods for genomic evaluation in pigs. Animal 6:1565-1571. https://doi.org/10.1017/S1751731112000742.

Gao, H., O. F. Christensen, P. Madsen, U. S. Nielsen, Y. Zhang, M. S. Lund, and G. Su. 2012. Comparison on genomic predictions using three GBLUP methods and two single-step blending methods in the Nordic Holstein population. Genet. Sel. Evol. 44:8. https://doi .org/10.1186/1297-9686-44-8

Gao, H., P. Madsen, U. S. Nielsen, G. P. Aamand, G. Su, K. Byskov, and J. Jensen. 2015. Including different groups of genotyped females for genomic prediction in a Nordic Jersey population. J. Dairy Sci. 98:9051-9059. https://doi.org/10.3168/jds.2015-9947.

García-Ruiz, A., J. B. Cole, P. M. VanRaden, G. R. Wiggans, F. J. Ruiz-López, and C. P. Van Tassell. 2016. Changes in genetic selection differentials and generation intervals in US Holstein dairy cattle as a result of genomic selection. Proc. Natl. Acad. Sci. USA 113:E3995-E4004. https://doi.org/10.1073/pnas.1519061113.

Garrick, D. J., J. F. Taylor, and R. L. Fernando. 2009. Deregressing estimated breeding values and weighting information for genomic regression analyses. Genet. Sel. Evol. 41:55. https://doi.org/10 .1186/1297-9686-41-55

Goddard, M. 2009. Genomic selection: Prediction of accuracy and maximisation of long term response. Genetica 136:245-257. https://doi.org/10.1007/s10709-008-9308-0.

Hayes, B. J., P. J. Bowman, A. J. Chamberlain, and M. E. Goddard. 2009. Invited review: Genomic selection in dairy cattle: Progress and challenges. J. Dairy Sci. 92:433-443. https://doi.org/10.3168/ jds.2008-1646.

Hozé, C., S. Fritz, F. Phocas, D. Boichard, V. Ducrocq, and P. Croiseau. 2014. Efficiency of multi-breed genomic selection for dairy cattle breeds with different sizes of reference population. J. Dairy Sci. 97:3918-3929. https://doi.org/10.3168/jds.2013-7761.

Jairath, L., J. C. M. Dekkers, L. R. Schaeffer, Z. Liu, E. B. Burnside, and B. Kolstad. 1998. Genetic evaluation for herd life in Canada. J. Dairy Sci. 81:550-562.

Jamrozik, J., and G. J. Kistemaker. 2016. Updated genetic parameters for Holstein reproductive traits using more recent data. Research Report to Genetic Evaluation Board, March 2016. pp. 9. Canadian Dairy Network, Guelph, Canada. https://www.cdn.ca/Articles/ GEBAPR2016/3_New\%20RP\%20Parameters\%20-\%20Janusz.pdf.

Legarra, A., O. F. Christensen, I. Aguilar, and I. Misztal. 2014. Single step, a general approach for genomic selection. Livest. Sci. 166:5465. https://doi.org/10.1016/j.livsci.2014.04.029.

Liu, Z., F. R. Seefried, F. Reinhardt, S. Rensing, G. Thaller, and R. Reents. 2011. Impacts of both reference population size and inclusion of a residual polygenic effect on the accuracy of genomic prediction. Genet. Sel. Evol. 43:19. https://doi.org/10.1186/1297 $-9686-43-19$

Lourenco, D. A L., I. Misztal, S. Tsuruta, I. Aguilar, E. Ezra, M. Ron, A. Shirak, and J. I. Weller. 2014a. Methods for genomic evaluation of a relatively small genotyped dairy population and effect of genotyped cow information in multiparity analyses. J. Dairy Sci. 97:1742-1752. https://doi.org/10.3168/jds.2013-6916. 
Lourenco, D. A. L., I. Misztal, S. Tsuruta, I. Aguilar, T. J. Lawlor, S. Forni, and J. I. Weller. 2014b. Are evaluations on young genotyped animals benefiting from the past generations? J. Dairy Sci. 97:3930-3942. https://doi.org/10.3168/jds.2013-7769.

Ma, P., M. S. Lund, U. S. Nielsen, G. P. Aamand, and G. Su. 2015. Single-step genomic model improved reliability and reduced the bias of genomic predictions in Danish Jersey. J. Dairy Sci. 98:9026-9034. https://doi.org/10.3168/jds.2015-9703.

Misztal, I., S. E. Aggrey, and W. M. Muir. 2013. Experiences with a single-step genome evaluation. Poult. Sci. 92:2530-2534. https:// doi.org/10.3382/ps.2012-02739.

Misztal, I., I. Aguilar, A. Legarra, and T. J. Lawlor. 2010. Choice of parameters for single-step genomic evaluation for type. J. Dairy Sci. 93(Suppl. 1):533. (Abstr.)

Misztal, I., H. L. Bradford, D. A. L. Lourenco, S. Tsuruta, Y. Masuda, and A. Legarra. 2017. Studies on inflation of GEBV in single-step GBLUP for type. Interbull Bull. 51:38-42.

Misztal, I., A. Legarra, and I. Aguilar. 2009. Computing procedures for genetic evaluation including phenotypic, full pedigree, and genomic information. J. Dairy Sci. 92:4648-4655. https://doi.org/10 $.3168 /$ jds.2009-2064.

Misztal, I., S. Tsuruta, T. Strabel, B. Auvray, T. Druet, and D. Lee 2002. BLUPF90 and related programs (BGF90). Communication No. 28-07 in Proc. 7th World Congr. Genet. Appl. Livest. Prod., Montpellier, France.

Patry, C., and V. Ducrocq. 2009. Bias due to genomic selection. Interbull Bull. 39:167-171. Uppsala, Sweden.

Patry, C., and V. Ducrocq. 2011. Evidence of biases in genetic evaluations due to genomic preselection in dairy cattle. J. Dairy Sci. 94:1011-1020. https://doi.org/10.3168/jds.2010-3804.

Sargolzaei, M., J. P. Chesnais, and F. S. Schenkel. 2014. A new approach for efficient genotype imputation using information from relatives. BMC Genomics 15:478. https://doi.org/10.1186/1471 $-2164-15-478$

Sargolzaei, M., F. S. Schenkel, and P. M. VanRaden. 2009. gebv: Genomic breeding value estimator for livestock. Technical report to the Dairy Cattle Breeding and Genetics Committee. University of Guelph, Guelph, ON, Canada.

Sorensen, D. A., and B. W. Kennedy. 1984. Estimation of response to selection using least squares and mixed model methodology. J. Anim. Sci. 58:1097-1106

Su, G., P. Madsen, U. S. Nielsen, E. A. Mäntysaari, G. P. Aamand, O. F. Christensen, and M. S. Lund. 2012. Genomic prediction for Nordic Red Cattle using one-step and selection index blending. J. Dairy Sci. 95:909-917. https://doi.org/10.3168/jds.2011-4804.

Sullivan, P. G. 2009. Options for combining direct genomic and progeny test results. Report to the Genetic Evaluation Board of CDN,
June 24, 2009 (unpublished). Canadian Dairy Network (CDN), Guelph, Ontario, Canada, www.cdn.ca.

Tsuruta, S., I. Misztal, I. Aguilar, and T. J. Lawlor. 2011. Multipletrait genomic evaluation of linear type traits using genomic and phenotypic data in US Holsteins. J. Dairy Sci. 94:4198-4204.

Tsuruta, S., I. Misztal, and T. J. Lawlor. 2013. Short communication: Genomic evaluations of final score for US Holsteins benefit from the inclusion of genotypes on cows. J. Dairy Sci. 96:3332-3335. https://doi.org/10.3168/jds.2012-6272.

Tsuruta, S., I. Misztal, and I. Stranden. 2001. Use of the preconditioned conjugate gradient algorithm as a generic solver for mixedmodel equations in animal breeding applications. J. Anim. Sci. 79:1166-1172

Uemoto, Y., T. Osawa, and J. Saburi. 2017. Effect of genotyped cows in the reference population on the genomic evaluation of Holstein cattle. Animal 11:382-393. https://doi.org/10.1017/ S1751731116001762.

Van Doormaal, B. J., G. J. Kistemaker, P. G. Sullivan, M. Sargolzaei, and F. Schenkel. 2009. Canadian implementation of genomic evaluations. Report to Interbull Open Meeting. Barcelona, Spain, August 21-24, 2009. Interbull Bull. 40:214-218.

VanRaden, P. M., C. Van Tassell, G. Wiggans, T. Sonstegard, R. Schnabel, J. Taylor, and F. Schenkel. 2009. Invited review: Reliability of genomic predictions for North American Holstein bulls. J. Dairy Sci. 92:16-24. https://doi.org/10.3168/jds.2008-1514.

VanRaden, P. M. 2008. Efficient methods to compute genomic predictions. J. Dairy Sci. 91:4414-4423. https://doi.org/10.3168/jds .2007-0980.

Vitezica, Z. G., I. Aguilar, I. Misztal, and A. Legarra. 2011. Bias in genomic predictions for populations under selection. Genet. Res. (Camb.) 93:357-366. https://doi.org/10.1017/ S001667231100022X.

Wiggans, G. R., T. A. Cooper, P. M. Vanraden, and J. B. Cole. 2011 Technical note: Adjustment of traditional cow evaluations to improve accuracy of genomic predictions. J. Dairy Sci. 94:6188-6193. https://doi.org/10.3168/jds.2011-4481.

Wiggans, G. R., J. B. Cole, S. M. Hubbard, and T. S. Sonstegard. 2017. Genomic selection in dairy cattle: The USDA experience. Annu. Rev. Anim. Biosci. 5:309-327. https://doi.org/10.1146/ annurev-animal-021815-111422.

Wiggans, G. R., P. M. Vanraden, and T. A. Cooper. 2012. Technical note: Adjustment of all cow evaluations for yield traits to be comparable with bull evaluations. J. Dairy Sci. 95:3444-3447. https:/ doi.org/10.3168/jds.2011-5000. 